\title{
Inhomogeneous vortex tangles in counterflow superfluid turbulence: flow in convergent channels
}

\author{
Lidia Saluto $^{1^{*}}$, Maria Stella Mongiovi ${ }^{2}$ \\ ${ }^{1}$ Dipartimento di Energia, Ingegneria dell'Informazione \\ e Modelli Matematici (DEIM) \\ Università degli Studi di Palermo, Palermo, Italy \\ ${ }^{2}$ Dipartimento di Ingegneria Chimica, Gestionale, \\ Informatica, Meccanica (DICGIM) \\ Università degli Studi di Palermo, Palermo, Italy \\ *Email address for correspondence: m.stella.mongiovi@unipa.it \\ Communicated by Vito Antonio Cimmelli and David Jou \\ Received on December 31, 2014. Accepted on June 17, 2015.
}

\begin{abstract}
We investigate the evolution equation for the average vortex length per unit volume $L$ of superfluid turbulence in inhomogeneous flows. Inhomogeneities in line density $L$ and in counterflow velocity $\mathbf{V}$ may contribute to vortex diffusion, vortex formation and vortex destruction. We explore two different families of contributions: those arising from a second order expansion of the Vinen equation itself, and those which are not related to the original Vinen equation but must be stated by adding to it second-order terms obtained from dimensional analysis or other physical arguments.

Keywords: Quantum turbulence, quantized vortices, heat transfer, inhomogeneous vortex tangle, vortex diffusion.

AMS subject classification: 82D50, 80A20, 76Fxx
\end{abstract}

\section{Introduction}

Liquid ${ }^{4}$ He has been extensively studied both from a theoretical and an experimental point of view, because of its unique properties, due to its quantum nature. An example of these peculiar features refers to heat transfer in counterflow experiments, characterized by no net matter flow, but only heat transport. If the heat flux is lower than a critical value $q_{c}$, the temperature gradient is so small that it is inappreciable to measurement, so indicating that the liquid has an extremely high thermal conductivity. This effect explains the remarkable ability of helium II to remove heat and makes it important in engineering applications: in fact, liquid helium is often used in the aerospace industry as refrigerant or for the refrigeration of large magnets [1]. 


\section{Inhomogeneous quantum vortex tangles}

A first important issue to be addressed in the problems of cryogenic refrigeration is the onset of turbulence. The superfluid turbulence can be generated thermally, applying a heat flux as in counterflow situation, or mechanically. As proved by $[2,3]$ in the turbulence generated mechanically, the energy spectrum is like the Kolmogorov spectrum, while in thermal counterflow is not so. In [4] some difference between the two kinds of turbulence are pointed out. Here we will consider thermally generated superfluid turbulence. Indeed, the presence of a sufficiently high heat flow causes the formation of a tangle of quantized vortex lines, which form, move, collide, and rearrange inside the superfluid [5-9]. The quantum of circulation is $\kappa$, given by $\kappa=h / m_{4}$, with $h$ the Planck constant, and $m_{4}$ the mass of ${ }^{4} \mathrm{He}$ atom. The tangle is usually assumed to be homogeneous and isotropic. This simplifies the mathematical analysis, as it makes possible to describe it in terms of a single scalar quantity $L$, the "vortex line density," i.e. the length of vortex lines per unit volume, having units of (length $)^{-2}$. The evolution equation for $L$ under constant values of the average counterflow velocity $V=|\mathbf{V}|$, with $\mathbf{V}=\left\langle\mathbf{v}_{n}-\mathbf{v}_{s}\right\rangle$, (being $\mathbf{v}_{n}$ and $\mathbf{v}_{s}$ the velocities of the normal and superfluid components), in the simplest case (absence of inhomogeneities and of rotation), was proposed by Vinen [10] by combining dimensional analysis and physical arguments. It is:

$$
\frac{d L}{d t}=\alpha_{V} V L^{\frac{3}{2}}-\beta_{V} \kappa L^{2},
$$

with $\alpha_{V}$ and $\beta_{V}$ dimensionless coefficients.

However, experiments [11-15], and numerical results [9,16-18] lead to consider inhomogeneous and anisotropic tangles, with special emphasis on the role of vortex diffusion. Here we will consider inhomogeneous situations, where the heat flux $\mathbf{q}$ (and consequently $L$ ) is inhomogeneous. In particular we explore the influence of $\nabla \mathbf{q}$ and $\nabla L$ on the evolution equation for $L$.

The results may be especially relevant in studies of strongly inhomogeneous flows, as for instance radial flows, flows in convergent or divergent channels [11-14], in the diffusion contribution to the decay of vortex tangles in narrow channels [18], or in entrance regions in channels. The results will be useful also in the study of mechanically generated turbulence. Indeed in [4] the authors observe that the vortex configuration is rather homogeneous for thermally driven turbulence and inhomogeneous for mechanically driven turbulence because of the spatial distribution.

In this paper we consider inhomogeneous situations, where the tangle may still be described by $L$, but $L$ may change from point to point in the volume of the tangle and an evolution equation for it must be written, taking explicitly into account the contributions of inhomogeneity, either as a 


\section{Saluto, M.S. Mongiovì}

diffusion flux of vortices $[7-9,18]$, or as an additional contribution to vortex formation or destruction, which could arise in convergent or divergent channels, as it was observed in some early pioneering but relatively forgotten experiments some years ago [11-15].

Indeed, much of the research on superfluid counterflow has been carried out along homogeneous channels, having the same cross section area along the whole channel. In this case, the inhomogeneities are radial, related to the presence of the walls, but they are very difficult to consider in narrow channels. Instead, convergent or divergent channels, or radial heat flux between concentric cylinders, offer a good opportunity to experimental research.

Some of the observations in convergent channels were described in terms of Geurst hydrodynamic model with an evolution equation for $L$ [19-21], given by:

(2) $\frac{\partial L}{\partial t}+\nabla \cdot\left[L V_{L}\right]+\kappa \frac{\gamma_{1}}{\gamma}\left[\frac{2}{L}|\nabla L|^{2}-\nabla^{2} L\right]=\frac{\beta}{2 \gamma} L^{\frac{3}{2}}\left|V_{L}-V\right|-\frac{\beta_{s}}{\gamma} L^{2}$,

with $\gamma_{1}, \gamma, \beta$ and $\beta_{s}$ dimensionless parameters.

The right-hand side of this equation corresponds to the classical Vinen's creation and destruction terms. In the left-hand side of Eq. (2), the second term corresponds to the convective term of the tangle moving at the local tangle velocity $V_{L}$, and the second contribution of the last term corresponds to the vortex diffusion, with $\kappa \frac{\gamma_{1}}{\gamma}$ being the diffusion coefficient; the first contribution to the last term indicates a non-local contribution to the vortex destruction, independent of the direction of the flow.

Along the present paper we will consider several equations incorporating diffusion and other non local effects. Instead of sticking only with Eq. (2) we will also study other possibilities.

In Section 2 we introduce a new term accounting for the role of the gradient of $\mathbf{V}$ (or of the heat flux $\mathbf{q}$ ) in vortex production, and we explore on the influence of it and of vortex diffusion in radial heat flows and in the entrance region. In Section 3, a nonlocal formulation of vortex line density evolution equation will be obtained adding to Vinen equation two kinds of new non-local terms: the firsts are obtained from a second-order Taylor expansion, in terms of the average vortex separation, given by $L^{-1 / 2}$; other second-order terms are added by using a dimensional analysis.

In Section 4, the concrete form of such equations for convergent (or divergent) channels will be specified and a set of physical conclusions will be driven. Final remarks are made in the concluding section. 


\section{Inhomogeneous quantum vortex tangles}

\section{Analysis of radial flow.}

Along the paper we explore evolution equations for $L$ in inhomogeneous situations. We concentrate our attention on dynamical effects, whereas in $[22,23]$ we considered this problem from a thermodynamic point of view, but with less diversity of dynamical equations than in the present paper. In this section we consider the following evolution equation instead of (2):

(3) $\frac{\partial L}{\partial t}+\nabla \cdot\left[L V_{L}\right]=\gamma^{\prime} q L^{\frac{3}{2}}-\gamma^{\prime \prime} L^{2}+D \nabla^{2} L+\alpha^{\prime} \mathcal{F}(\nabla \mathbf{q}) L+\alpha^{\prime \prime} \mathbf{q} \cdot \nabla L$,

where $\gamma^{\prime}, \gamma^{\prime \prime}, D, \alpha^{\prime}$ and $\alpha^{\prime \prime}$ are coefficients with suitable dimensions (in particular $\gamma^{\prime}=\alpha_{V} /\left(\rho_{s} T s\right)$ and $\left.\gamma^{\prime \prime}=\beta_{V} \kappa\right)$, while $\mathcal{F}(\nabla \mathbf{q})$ is a scalar functional of $\nabla \mathbf{q}$, as for instance $[(\nabla \mathbf{q}):(\nabla \mathbf{q})]^{\frac{1}{2}}$ or $\hat{\mathbf{u}} \cdot(\nabla \mathbf{q}) \cdot \hat{\mathbf{u}}$, with $\hat{\mathbf{u}}$ the unit vector in a given direction, i.e. that of the heat flux $\mathbf{q}$ or that of the Schwarz vector I, a characteristic vector introduced by Schwarz [24], defined as

$$
\mathbf{I} \equiv \frac{\int \mathbf{s}^{\prime} \times \mathbf{s}^{\prime \prime} d \xi}{\int\left|\mathbf{s}^{\prime \prime}\right| d \xi}
$$

where $\mathbf{s}^{\prime}$ is the unit vector tangent to the vortex line described by $\mathbf{s}(\xi)$, with $\xi$ the arc-length, and $\mathbf{s}^{\prime \prime}$ the curvature of the vortex line. As it is known, in Schwarz derivation of Vinen equation, the first term in the right hand size of equation (3) is substituted with $\gamma \mathbf{q} \cdot \mathbf{I} L^{\frac{3}{2}}=\gamma I_{0} q L^{\frac{3}{2}}$ [24].

The diffusion term in $D$ and the term in $\alpha^{\prime \prime}$ were considered by us in $[22,23,25]$ from a thermodynamic point of view. The original proposal of this section is the term in $\alpha^{\prime}$, that describes the role of the heat gradient as vortex source. Other terms will be considered in the next section. In the well known Vinen equation, the vortex source is considered to be related to heat flux modulus $|\mathbf{q}|=q$, but independent of heat gradients (which in the two fluid model are related to gradients of the counterflow velocity $\mathbf{V}$ ). However the heat flux gradients are expected to act as natural source of vorticity, and therefore of vortex lines, in addition to the usual term in $\gamma^{\prime}$.

We will separately consider the effect of the diffusion term in $D$ (which were already studied in [23]), the term in $\alpha^{\prime \prime}$, that is considered here from a new perspective and $\alpha^{\prime}$, which is considered here for the first time. To this purpose we apply equations (3) to a steady-state counterflow of $\mathrm{He}$ II between two concentric cylindrical walls at different temperatures as studied in [26], where the author analyzes a rotating annulus driven by radial counterflow in a two dimensional setting.

The situation considered here has a relative mathematical simplicity as compared with the more usual counterflow along a cylindrical container studied in [27-29] (see Figure 1-left). The inner cylinder (the hotter one) is 


\section{Saluto, M.S. Mongiovì}

at fixed temperature $T_{0}$, the temperature profile $T(r)$ between both cylinders will depend on the heat flux, the thermal conductivity, and the vortex line density profile $L(r)$. Since in this situation the heat flow has only a radial component, which depends only on the radial coordinate, namely $q(r)$, this situation is the simplest one with a heat gradient.
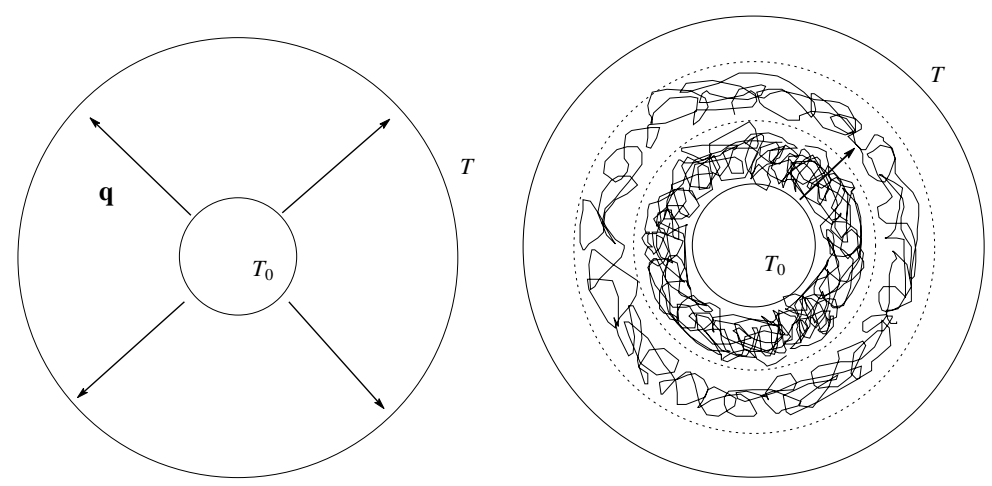

Figure 1. Left: Heat flows axially from the inner cylinder (the hotter one), at fixed temperature $T_{0}$, to the outer colder one. The radial temperature profile $T(r)$ depends on the heat flow and the vortex density radial distribution (from [23]). Right: Example of vortex diffusion. When the diffusion plays a role one can observe simultaneously several regimes of turbulence, in the same experiment (close to the inner cylinder the vortex production is higher, then one can observe a diffusive flow of vortices).

\subsection{Diffusion contribution.}

The steady-state situation requires $\nabla \cdot \mathbf{q}=0$ according to the energy balance equation. We can consider axial symmetry for the problem, hence, the heat flux has only the radial component $q_{r}$ (which will be called here as $q$ ). This implies that $q=\Gamma / r$, with $\Gamma$ being the heat supplied per unit time and unit length of the cylinder and $r$ the distance with respect to the axis of the cylinders.

Here we briefly recall our results for the behavior of $L(r)$ [23], which could be obtained experimentally from the attenuation coefficient of second sound. Introduction of $q=\Gamma / r$ in (3), and ignoring, for the moment, the terms in $\alpha^{\prime}$ and in $\alpha^{\prime \prime}$ (which will be considered in Section 2.2), allows us to obtain $L(r)$ from:

$$
\gamma^{\prime} \frac{\Gamma}{r} L^{\frac{3}{2}}-\gamma^{\prime \prime} L^{2}+D \nabla^{2} L=0
$$

If the temperature gradients are small, $\gamma^{\prime}, \gamma^{\prime \prime}$ and $D$ (which in principle depend on temperature) may be taken as constant. Equation (5) admits 


\section{Inhomogeneous quantum vortex tangles}

the solution:

$$
L(r)=\bar{\alpha}^{2} \frac{\Gamma^{2}}{r^{2}},
$$

with

$$
\bar{\alpha}=\frac{\gamma^{\prime}}{2 \gamma^{\prime \prime}}\left(1+\sqrt{1+\frac{16 D \gamma^{\prime \prime}}{\gamma^{\prime 2} \Gamma^{2}}}\right) .
$$

If $D=0$ one recovers the result $\bar{\alpha}=\gamma^{\prime} / \gamma^{\prime \prime}$, and according to the usual Vinen equation:

$$
L(r)=\frac{\gamma^{\prime 2}}{\gamma^{\prime \prime 2}} \frac{\Gamma^{2}}{r^{2}} .
$$

Instead, when the diffusion effects become dominating, $L(r)$ takes the form:

$$
L(r)=\frac{4 D}{\gamma^{\prime \prime} r^{2}} .
$$

In this regime, $L$ is independent of the heat flux, but it keeps the same dependence $1 / r^{2}$ on the radius than in (6) for $D=0$. Thus, the influence of the vortex diffusion is focused on the local values of $L(r)$ rather than on the form of the spatial steady distribution.

It is seen in (7) that the diffusion increases the local values of $L$ as compared with the situation with $D=0$, especially at relatively low values of the heat flux. This increase may be intuitively understood as the result of a diffusive flow of vortices from the hotter cylinder (where $\mathbf{q}$ is higher and therefore the vortex production is higher) to the colder cylinder, where they will disappear when colliding against the external wall (see Figure 1right). Thus, the term in $\gamma^{\prime} \Gamma$ accounts for the vortices produced in the same point being considered, in contrast with the term in $D$, which accounts for the "migrating" vortices in that point. For small heat flux or high diffusion, the "migrating" vortex population is higher than the "native" vortex population.

The result (6) of the different values of $L$ in the presence or absence of diffusion suggests the possibility of hysteresis when the heat flux is increased from zero to some maximum value, at a rate sufficiently higher than the diffusion rate, and is subsequently lowered to zero, at a rate sufficiently lower than the diffusion rate. In the first case, diffusion will not have time to act during the process, so that $L$ will be given by (6)-(7) with $D=0$ (the "native" population will predominate), i.e. $L(r)$ given by equation (8). 


\section{Saluto, M.S. Mongiovì}

In contrast, in the slow process, diffusion will always act and $L$ will be described by $(6)-(7)$ with $D \neq 0$. We have commented this aspect with detail in [23] and we summarize it in Figure 2, where we have plotted the dimensionless quantity $L r^{2}$ in terms of the dimensionless heat flux $\alpha_{0} \Gamma$, with $\alpha_{0} \equiv \frac{\gamma^{\prime}}{2 \gamma^{\prime \prime}}$.

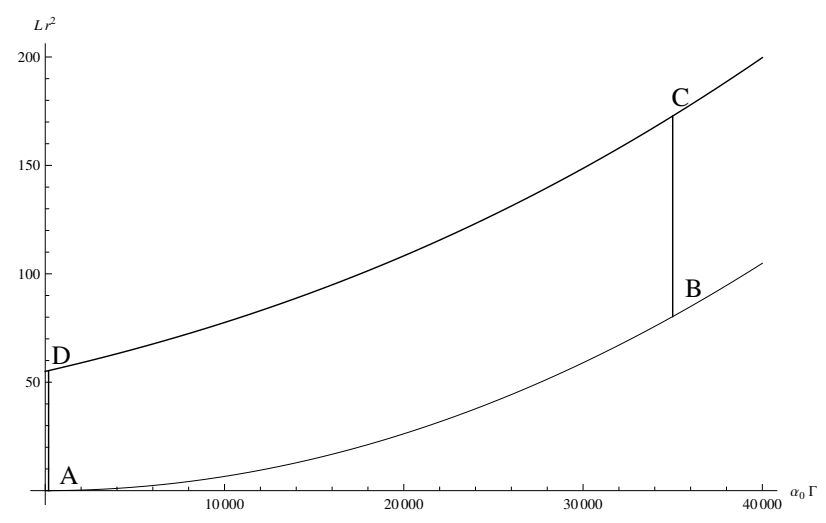

Figure 2. Hysteresis cycle. The value of $L r^{2}$ (vertical axis) as a function of the dimensionless expression for the heat flux $\alpha_{0} \Gamma$, with $\alpha_{0} \equiv \frac{\gamma^{\prime}}{2 \gamma^{\prime \prime}}$. The value of $L$ for fastly increasing heat flux (A to B), corresponding to eq. (8), is different from that for slowly decreasing heat flux (C to $\mathrm{D})$, corresponding to eq. (6)-(7) with $D \neq 0$. The values of $\alpha_{0}=1.28 * 10^{-4} \mathrm{~s}^{3} /\left(\mathrm{cm}^{*} \mathrm{~g}\right)$ for $T=1.5 \mathrm{~K}$ and $D=2.2 \kappa \mathrm{cm}^{2} / \mathrm{s}$ have been taken from [16] for $D$, from [30] for $\gamma^{\prime \prime}$, and from [31] for the ratio $\gamma^{\prime} / \gamma^{\prime \prime}$.

\subsection{Heat gradient contribution.}

In the radial heat flow, the only non-vanishing term of the heat flux gradient is $d q / d r$. In this case if one chooses the expression $[(\nabla \mathbf{q}):(\nabla \mathbf{q})]^{\frac{1}{2}}$ for $\mathcal{F}(\nabla \mathbf{q})$, we get $\alpha^{\prime} \mathcal{F}(\nabla \mathbf{q})=\alpha^{\prime}|d q / d r|$, while if one chooses for $\mathcal{F}(\nabla \mathbf{q})$ the form $\hat{\mathbf{r}} \cdot(\nabla \mathbf{q}) \cdot \hat{\mathbf{r}}$, with $\hat{\mathbf{r}}$ the unit vector in the radial direction, we get $\alpha^{\prime} \mathcal{F}(\nabla \mathbf{q})=\alpha^{\prime} \nabla \mathbf{q} \cdot \hat{\mathbf{r}}= \pm \alpha^{\prime}|d q / d r|$. In the first case the contribution of this term has constant sign, in the second its contribute depends on the direction of the radial heat flux.

If the radial gradient of $\mathbf{q}$, i.e. the term in $\alpha^{\prime}$ in (3), contributes as an additional positive source of vortices $\left(\alpha^{\prime}>0\right)$, but the effects of $D$ are neglected, we have for the steady-state vortex length:

$$
\gamma^{\prime \prime} L-\gamma^{\prime} q L^{\frac{1}{2}}-\alpha^{\prime}\left|\frac{d q}{d r}\right|=0 .
$$

Since $q=\Gamma / r,|d q / d r|=\Gamma / r^{2}$, and $L(r)$ is given by (6), with $\bar{\alpha}$ given 


\section{Inhomogeneous quantum vortex tangles}

by:

$$
\bar{\alpha}=\frac{\gamma^{\prime}}{2 \gamma^{\prime \prime}}\left[1+\sqrt{1+\frac{4 \gamma^{\prime \prime} \alpha^{\prime}}{\gamma^{\prime 2} \Gamma}}\right],
$$

instead of (7). Thus, the dependence of $L$ with the radius would be the same as for $\alpha^{\prime} \neq 0$, but the coefficient would be different, implying a higher value of $L$, because the gradient of $\mathbf{q}$ acts as an additional source of vortices. Note that the extra term under the square root in (11) is proportional to $\alpha^{\prime} / \Gamma$ whereas it is proportional to $D / \Gamma^{2}$ in (7), with $\Gamma$ related to $q(r)$ as $q(r)=\Gamma / r$, as it has just been said. Thus, a possible way of comparing whether the increase in $L(r)$ in axial flow is due to $|d q / d r|$ as a source, or if it is due to the diffusion term $D \nabla^{2} L$, is to see the dependence of the coefficient $\bar{\alpha}$ in (11) by $\Gamma$ (as $\Gamma^{-1}$ or as $\Gamma^{-2}$ respectively) whereas the influence on $1 / r^{2}$ is the same one for both.

\subsection{Heat gradient and entrance region.}

Another way to deal with the relative directionality of the heat flux on the vortex productions is through the term $\alpha^{\prime \prime} \mathbf{q} \cdot \nabla L$ in (3). Since in a first approximation $L$ is proportional to $q^{2}$, the term $\alpha^{\prime \prime} \mathbf{q} \cdot \nabla L$ will have different sign if $\mathbf{q}$ points to region with increasing values of $\mathbf{q}$ or with decreasing values of $\mathbf{q}$. Since in axial flows $|\mathbf{q}|$ is higher in central regions, and also $L$, one has $\mathbf{q} \cdot \nabla L>0$ for $\mathbf{q}$ going toward the center and $\mathbf{q} \cdot \nabla L<0$ for $\mathbf{q}$ going away from the center (see Figure 3).

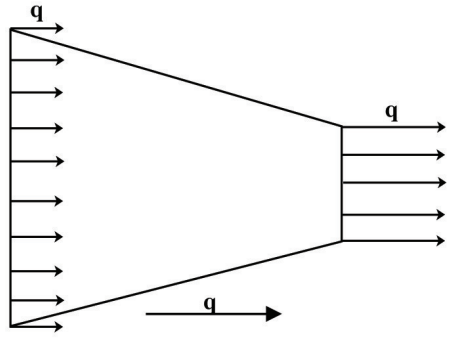

(a) $\mathbf{q} \cdot \nabla \mathrm{L}>0$

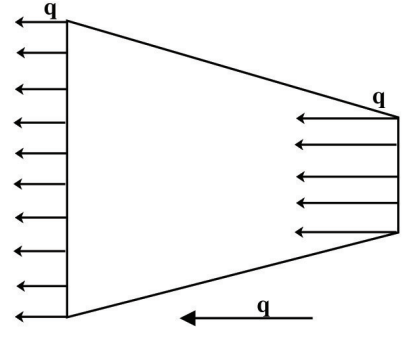

(b) $\mathbf{q} \cdot \nabla \mathrm{L}<0$

Figure 3. Longitudinal section of a channel with heat flow going from wide to narrow regions (a), and opposite (b). In (a) $\mathbf{q} \cdot \nabla L>0$; in (b) $\mathbf{q} \cdot \nabla L<0$. In the classical local form of Vinen equation, this difference in $\mathbf{q} \cdot \nabla L$ does not influence the local value of the vortex length density $L$; in contrast, equation (10) in our model leads to different values for the local values of $L$ in both situations. 


\section{Saluto, M.S. Mongiovì}

This means that if the left wall is at temperature $T_{H}$ (hotter temperature) and the right one at $T_{C}$ (colder temperature), the direction of the heat flux is opposite with respect to the reverse situation, in which the left wall is at temperature $T_{C}$ and the other at $T_{H}$. Thus this would contribute in a natural way to heat rectification, but with quantitative effects depending on the value of $\alpha^{\prime \prime}$. Thermal rectification is an interesting phenomenon in nanotechnologies, in which thermal transport along a specific axis is dependent on the sign of the temperature gradient, and in superfluid helium it can be important in refrigeration problems.

Another situation where the influence of heat gradients may be relevant could be the region of entrance flows in cylindrical or flat channels.

The entrance region is that region from the origin of the channel $(x=0$, with $x$ the direction of the axis of the channel), where the heat flux may be given with an arbitrary form (usually considered as a flat profile), to the positions where the heat flux distribution has become independent of $x$. This region is a classical but very difficult topic of fluid mechanics heat transport.

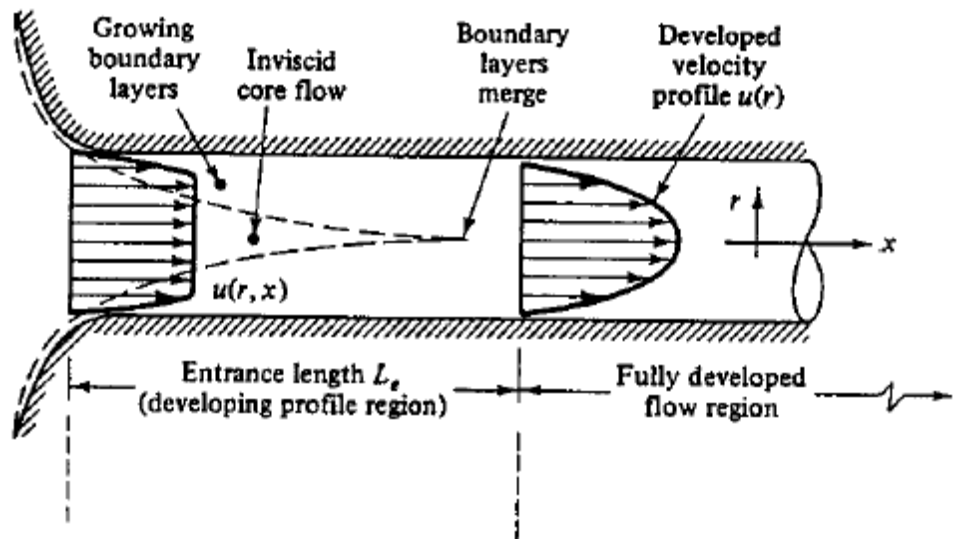

Figure 4. Developing velocity profile in the entrance of a duct flow. One can see the entrance region, where the velocity profile evolves from flat to parabolic.

In the fully developed region along a right cylindrical channel, the heat flux will have only the longitudinal component $q_{x}=q(r)$. However, in the entrance region, where $q_{x}=q(x, r)$ depends on $x$, one will have $\partial q_{x} / \partial x \neq 0$. Furthermore, because of the steady-state condition $\nabla \cdot \mathbf{q}=0$, which leads 


\section{Inhomogeneous quantum vortex tangles}

to:

$$
\frac{1}{r} \frac{\partial}{\partial r}\left(r q_{r}\right)+\frac{\partial q_{x}}{\partial x}=0 .
$$

The fact that $\partial q_{x} / \partial x \neq 0$ does in turn imply that $\partial\left(r q_{r}\right) / \partial r \neq 0$, i.e. that the radial component $q_{r}(x, r)$ cannot be ignored. We will further have $\partial q_{r} / \partial x \neq 0$. Thus, if the term in $\alpha^{\prime \prime}$ in (3) is positive, it would follow that this term would strongly contribute to vortex formation already at the origin of the entrance regions.

\section{Non-local extensions of Vinen equation.}

In this section we consider several different ways of generalizing SchwarzVinen equation with diffusion effects, namely

$$
\frac{d L}{d t}=\tilde{\alpha} \mathbf{V} \cdot \mathbf{I} L^{\frac{3}{2}}-\beta_{V} \kappa L^{2}+D \nabla^{2} L
$$

in the presence of $\nabla \mathbf{V}$ and $\nabla L$. Here we use the counterflow velocity $\mathbf{V}$ rather than $\mathbf{q}$ (that has been used in (3)) because it is more well known, in the context of two-fluid model of turbulence. In the one-fluid model, using $\mathbf{q}$ instead of $\mathbf{V}$ seems more natural and more closely related to observational quantities. Anyway, since in counterflow $\mathbf{q}=\rho_{s} s T \mathbf{V}$, going from one formalism to the other is not difficult.

Diffusion effects are the most direct and intuitive non-local contribution to the dynamics of the vortices, but other non-local effects may appear, related to the production or destruction of vortices, as seen in (3). We will proceed our exploration in a systematic way. First, we will expand up to second order in $L^{-1 / 2}$ (the average distance among vortex lines) the production and destruction terms in Schwarz-Vinen equation. Second, we will incorporate other possible terms following from dimensional analysis.

We assume, for the sake of simplicity, that the effects of the temperature gradient are much less than the effects of the gradients of $\mathbf{V}$ and $L$, so that they can be neglected.

\subsection{Series expansion of the production and destruction terms.}

Taking into account that the average separation $\delta$ between vortex lines in the tangle is of the order of $L^{-\frac{1}{2}}$, it may be expected that the creation and destruction processes will be affected by inhomogeneities of $\mathbf{V}$ and $L$, because vortices from $x-\delta$ and from $x+\delta$ will collide with vortices at $x$, and, therefore, their effects should be considered, instead of considering only the interactions of vortices already at $x$. The non-local contributions 


\section{Saluto, M.S. Mongiovì}

will be specially relevant for small values of $L$, where the mean free path $\delta=L^{-\frac{1}{2}}$ is long.

The expansion up to second order in $d \mathbf{x}=L^{-1 / 2} \hat{\mathbf{u}}$ (with $\hat{\mathbf{u}}$ an arbitrary unit vector) of the creation term is given by:

$$
\begin{aligned}
& {\left[\frac{\partial L}{\partial t}\right]_{\text {prod }}=\tilde{\alpha}\left\{\mathbf{V} \cdot \mathbf{I} L^{\frac{3}{2}}+L \hat{\mathbf{u}} \cdot[(\nabla \mathbf{V}) \cdot \mathbf{I}+\mathbf{V} \cdot(\nabla \mathbf{I})]\right.} \\
& +\frac{1}{2} L^{\frac{1}{2}} \hat{\mathbf{u}} \cdot[(\nabla(\nabla \mathbf{V})) \cdot \mathbf{I}+2 \nabla \mathbf{V}: \nabla \mathbf{I}+\mathbf{V} \cdot(\nabla(\nabla \mathbf{I}))] \cdot \hat{\mathbf{u}} \\
& +(\mathbf{V} \cdot \mathbf{I})\left[\frac{3}{2} \hat{\mathbf{u}} \cdot \nabla L+\frac{3}{8} L^{-\frac{3}{2}}|\nabla L|^{2}+\frac{3}{4} L^{-\frac{1}{2}} \nabla^{2} L\right] \\
& \left.+\frac{3}{2} L^{-\frac{1}{2}}[\mathbf{I} \cdot(\nabla \mathbf{V}) \cdot \nabla L+\mathbf{V} \cdot(\nabla \mathbf{I}) \cdot \nabla L]\right\} .
\end{aligned}
$$

On the other side the destruction term is approximated by:

$$
\left[\frac{\partial L}{\partial t}\right]_{\text {destr }}=\beta_{V} \kappa\left\{L^{2}+2 L^{\frac{1}{2}} \hat{\mathbf{u}} \cdot \nabla L+L^{-1}|\nabla L|^{2}+\nabla^{2} L\right\}
$$

Thus, a non local extension of (13) is obtained substituting to SchwarzVinen original production and destruction terms the expressions (14) and (15).

Assuming $\mathbf{V}$ and $\mathbf{I}$ collinear and $\mathbf{I}$ constant, a non-local hydrodynamical extension of equation (13) would be:

$$
\begin{aligned}
& \frac{\partial L}{\partial t}=\tilde{\alpha} I\left[V L^{\frac{3}{2}}+L \hat{\mathbf{u}} \cdot(\nabla \mathbf{V}) \cdot \hat{\mathbf{u}}+\frac{1}{2} L^{\frac{1}{2}} \hat{\mathbf{u}} \cdot[\nabla(\nabla \mathbf{V})] \cdot \hat{\mathbf{u}}\right. \\
+ & \left.\frac{3}{2} V \hat{\mathbf{u}} \cdot \nabla L+V\left(\frac{3}{8} L^{-\frac{3}{2}}|\nabla L|^{2}+\frac{3}{4} L^{-\frac{1}{2}} \nabla^{2} L\right)+\frac{3}{2} L^{-\frac{1}{2}} \hat{\mathbf{u}} \cdot \nabla \mathbf{V} \cdot \nabla L\right] \\
- & \beta_{V} \kappa\left[L^{2}+2 L^{\frac{1}{2}} \hat{\mathbf{u}} \cdot \nabla L+L^{-1}|\nabla L|^{2}\right]+\left(D-\beta_{V} \kappa\right) \nabla^{2} L .
\end{aligned}
$$

Here, we want to consider situations where the heat flux has approximately only one component and depends only on one coordinate. This is the case of radial flows, in an exact sense, and of convergent channels in an approximate sense. In this simplified situation it is $\mathbf{V}=V \hat{\mathbf{x}}, \mathbf{I}=I \hat{\mathbf{x}}$, and 


\section{Inhomogeneous quantum vortex tangles}

the previous equation becomes:

$$
\begin{aligned}
\frac{\partial L}{\partial t} & =\tilde{\alpha} I\left\{V L^{\frac{3}{2}}+\frac{\partial V}{\partial x} L+\frac{1}{2} L^{\frac{1}{2}} \frac{\partial^{2} V}{\partial x^{2}}+\frac{3}{2} L^{-\frac{1}{2}} \frac{\partial V}{\partial x} \frac{\partial L}{\partial x}\right. \\
& \left.+V\left[\frac{3}{2} \frac{\partial L}{\partial x}+\frac{3}{8} L^{-\frac{3}{2}}\left(\frac{\partial L}{\partial x}\right)^{2}+\frac{3}{4} L^{-\frac{1}{2}} \frac{\partial^{2} L}{\partial x^{2}}\right]\right\} \\
& -\beta_{V} \kappa\left[L^{2}+2 L^{1 / 2} \frac{\partial L}{\partial x}+L^{-1}\left(\frac{\partial L}{\partial x}\right)^{2}\right]+\left(D-\beta_{V} \kappa\right) \frac{\partial^{2} L}{\partial x^{2}}
\end{aligned}
$$

Note that this equation contains two non-local terms formally similar to those which are present in Geurst model (2), but many other additional terms too.

Thus, equation (17) is a simple and direct implementation of an evolution of $L$ starting from Vinen equation, but other kinds of term not coming from such a non-local extension may also contribute, in principle, to such non-local extension.

\subsection{Dimensional analysis and additional non-local terms.}

On dimensional grounds one may directly incorporate to (13) terms depending on $\nabla L$ and on $\nabla \mathbf{V}$, and requiring that they have the correct physical dimensions. Later on, one must consider their physical meaning, their compatibility with the second law of thermodynamics, their agreement or lack of agreement with experimental observations, and their microscopic bases. Up to second order, a simple generalization is:

$$
\begin{aligned}
\frac{d L}{d t} & =\alpha_{V} V L^{\frac{3}{2}}-\beta_{V} \kappa L^{2}+D \nabla^{2} L-\gamma_{1} \mathbf{V} \cdot \nabla L \\
& -\gamma_{2} \frac{V^{2}}{\kappa L}|\nabla L|^{2}-\gamma_{3} L[(\nabla \mathbf{V}):(\nabla \mathbf{V})]^{\frac{1}{2}},
\end{aligned}
$$

where we have put $\alpha_{V}=\tilde{\alpha} I$, and where we have chosen the expression $\gamma_{3} L[(\nabla \mathbf{V}):(\nabla \mathbf{V})]^{\frac{1}{2}}$ for the functional $\mathcal{F}(\nabla \mathbf{V})$. This term will be a production term (if $\gamma_{3}<0$ ) or a destruction term (if $\gamma_{3}>0$ ) and it is due to the inhomogeneity in $\mathbf{V}$. When it is expressed in terms of $\mathbf{q}$, it plays a role analogous to the term in $\alpha^{\prime}$ in (3). The term in $\mathbf{V} \cdot \nabla L$, when expressed in terms of $\mathbf{q}$, plays a role analogous to the term in $\alpha^{\prime \prime}$ in (3). The term in $V^{2}|\nabla L|^{2}$ is new, indeed it does not appear in the extension (17) nor in the equation (3). Thus, taking in mind (16) and (18), a possible generalized 


\section{Saluto, M.S. Mongiovì}

non-local equation should be:

$$
\begin{aligned}
\frac{\partial L}{\partial t} & =\alpha_{V} V L^{\frac{3}{2}}-\beta_{V} \kappa L^{2}-2 \beta_{V} \kappa L^{\frac{1}{2}} \hat{\mathbf{u}} \cdot \nabla L \\
& +\left(D-\beta_{V} \kappa+\frac{3}{4} \alpha_{V} V L^{-\frac{1}{2}}\right) \nabla^{2} L \\
& -\left(\beta_{V} \kappa \frac{1}{L}-\gamma_{2} \frac{V^{2}}{\kappa L}+\frac{3}{8} \alpha_{V} V L^{-\frac{3}{2}}\right)|\nabla L|^{2} \\
& +\left(\frac{3}{2} \alpha_{V} V \hat{\mathbf{u}}-\gamma_{1} \mathbf{V}\right) \cdot \nabla L+\frac{3}{2} L^{-\frac{1}{2}} \hat{\mathbf{u}} \cdot \nabla \mathbf{V} \cdot \nabla L \\
& -\gamma_{3}[(\nabla \mathbf{V}):(\nabla \mathbf{V})]^{\frac{1}{2}} L+\alpha_{V} L \hat{\mathbf{u}} \cdot(\nabla \mathbf{V}) \cdot \hat{\mathbf{u}} \\
& +\frac{1}{2} \alpha_{V} L^{\frac{1}{2}} \hat{\mathbf{u}} \cdot[\nabla(\nabla \mathbf{V})] \cdot \hat{\mathbf{u}} .
\end{aligned}
$$

\section{Application to averaged one-dimensional description of con- vergent channels.}

The situation that we are considering here, is represented in Figure 5. A constant heat flow $\dot{Q}$ is imposed in the direction of $x$-axis, $r$ indicates the width of the channel and it depends on the position $x$, while the length $h$ of the channel is considered constant.

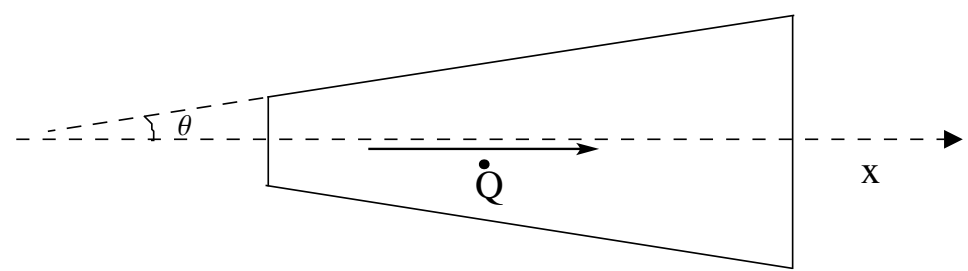

Figure 5. A constant heat $\dot{Q}$ is imposed in the $x$-direction. The channel has a constant length $h$, and a variable width $r(x)=\theta x$, where $\theta$ is the slope of the walls with respect to the axis of the channel.

In this situation, one has from the energy balance equation:

$$
q(x) h r(x)=A \dot{Q},
$$

with $A$ constant, and therefore:

$$
q(x)=\frac{A \dot{Q}}{h r(x)}=\frac{B^{\prime}}{r(x)},
$$




\section{Inhomogeneous quantum vortex tangles}

where $B^{\prime}=\frac{A \dot{Q}}{h}$ is constant. Because in counterflow it is $q=\rho_{s} T s V$ we obtain also:

$$
V(x)=\frac{B}{r(x)},
$$

where $B=B^{\prime} /\left(\rho_{s} T s\right)$.

In this case it is $\hat{\mathbf{u}}=\hat{\mathbf{x}}$ and, assuming that $\nabla \mathbf{V}$ is collinear with $\hat{\mathbf{x}}$, equation (19) is written:

$$
\begin{aligned}
\frac{\partial L}{\partial t} & =\alpha_{V} V L^{\frac{3}{2}}-\beta_{V} \kappa L^{2}+\left(\alpha_{V} \frac{\partial V}{\partial x}-\gamma_{3}\left|\frac{\partial V}{\partial x}\right|\right) L \\
& +\left[\left(\frac{3}{2} \alpha_{V}-\gamma_{1}\right) V-2 \beta_{V} \kappa L^{\frac{1}{2}}+\frac{3}{2} L^{-\frac{1}{2}} \frac{\partial V}{\partial x}\right] \frac{\partial L}{\partial x} \\
& -\left(\beta_{V} \kappa \frac{1}{L}-\gamma_{2} \frac{V^{2}}{\kappa L}+\frac{3}{8} \alpha_{V} V L^{-\frac{3}{2}}\right)\left|\frac{\partial L}{\partial x}\right|^{2} \\
& +\left(D-\beta_{V} \kappa+\frac{3}{4} \alpha_{V} V L^{-\frac{1}{2}}\right) \frac{\partial^{2} L}{\partial x^{2}}+\frac{1}{2} \alpha_{V} L^{\frac{1}{2}} \frac{\partial^{2} V}{\partial x^{2}} .
\end{aligned}
$$

Since in (23) there are many terms, in the following subsections we consider some of these additional terms one by one independently and then we propose the general expression for a local perturbation $L^{\prime}$ containing all the terms, in order to understand their physical consequences in a given physical situation, which is a necessary step to propose suitable experiments to check the equations. According with Vinen equation, the vortex line density $L$, at each point $x$, should be given by:

$$
L(x)=\left(\frac{\alpha_{V}}{\beta_{V} \kappa}\right)^{2} V^{2}(x),
$$

with the local counterflow velocity $V(x)$ dependent on $x$ as in (22). Local perturbations, due to the presence of these terms, with respect to Vinen's prediction shall be analyzed in detail. In particular, we will assume that the opening angle $\theta$ is relatively small, so that $\tan \theta$ can be approximated by $\theta$, and determine the several terms that must be added to the classical expression (24) for $L$, in terms of $\theta$ and $x$ (or $\theta$ and $r$ ).

\subsection{Only a term in $\partial L / \partial x$.}

We will start with a term in $\frac{\partial L}{\partial x}$ (ignoring all the other terms), as in the following expression:

$$
\frac{\partial L}{\partial t}=\alpha_{V} V L^{\frac{3}{2}}-\beta_{V} \kappa L^{2}-\gamma_{1} V \frac{\partial L}{\partial x} .
$$




\section{Saluto, M.S. Mongiovì}

In the steady-state, if one considers a perturbation $L^{\prime}$ of the homogeneous solution of (25), $L_{h}$ given by $L_{h}=\left(\alpha_{V} / \beta_{V} \kappa\right)^{2} V^{2}(x)$, namely $L=L_{h}(x)+L^{\prime}$, one would have, from the linearized version of $(25)$ :

$$
\frac{3}{2} \alpha_{V} V L_{h}^{\frac{1}{2}} L^{\prime}-2 \beta_{V} \kappa L_{h} L^{\prime}=\gamma_{1} V \frac{\partial L_{h}}{\partial x},
$$

where for the sake of simplicity we have assumed $L^{\prime}$ independent of $x$, and then, one obtains for $L^{\prime}$ :

$$
L^{\prime}=-2 \frac{\beta_{V} \kappa}{\alpha_{V}^{2} V^{2}} \gamma_{1} V \frac{\partial}{\partial x}\left[\left(\frac{\alpha_{V}}{\beta_{V} \kappa}\right)^{2} V^{2}\right]=-4 \frac{\gamma_{1}}{\beta_{V} \kappa} \frac{\partial V}{\partial x}=4 \frac{\gamma_{1}}{\beta_{V} \kappa} \frac{B \theta}{r^{2}} .
$$

4.2. Only a term in $\left|\frac{\partial L}{\partial x}\right|^{2}$.

A second contribution in order to modify Vinen equation can be the following:

$$
\frac{\partial L}{\partial t}=\alpha_{V} V L^{\frac{3}{2}}-\beta_{V} \kappa L^{2}+\gamma_{2} \frac{V^{2}}{\kappa L}\left|\frac{\partial L}{\partial x}\right|^{2} .
$$

In this case, one obtains:

$$
L^{\prime}=8 \frac{\gamma_{2}}{\beta_{V} \kappa^{2}}\left(\frac{\partial V}{\partial x}\right)^{2}=8 \frac{\gamma_{2}}{\beta_{V} \kappa^{2}} \frac{B^{2} \theta^{2}}{r^{4}} .
$$

4.3. Only a term in $\frac{\partial^{2} L}{\partial x^{2}}$.

Adding only a term proportional to $\frac{\partial^{2} L}{\partial x^{2}}$, one recovers:

$$
\frac{\partial L}{\partial t}=\alpha_{V} V L^{\frac{3}{2}}-\beta_{V} \kappa L^{2}+D \frac{\partial^{2} L}{\partial x^{2}} .
$$

In this case, one obtains:

$$
L^{\prime}=4 \frac{D}{\beta_{V} \kappa} \frac{1}{V^{2}}\left[\left(\frac{\partial V}{\partial x}\right)^{2}+V \frac{\partial^{2} V}{\partial x^{2}}\right]=12 \frac{D}{\beta_{V} \kappa} \frac{\theta^{2}}{r^{2}} .
$$

4.4. Only a term in $\frac{\partial V}{\partial x}$.

Choosing in equation only the term in $[\nabla \mathbf{V}: \nabla \mathbf{V}]^{\frac{1}{2}}$ (the term in $\hat{\mathbf{x}}$. $(\nabla \mathbf{V}) \cdot \hat{\mathbf{x}}$ leads to a opposite result), one has:

$$
\frac{\partial L}{\partial t}=\alpha_{V} V L^{\frac{3}{2}}-\beta_{V} \kappa L^{2}-\gamma_{3} L\left|\frac{\partial V}{\partial x}\right|
$$

and then:

$$
L^{\prime}=-2 \frac{\gamma_{3}}{\beta_{V} \kappa}\left|\frac{\partial V}{\partial x}\right|=-2 \frac{\gamma_{3}}{\beta_{V} \kappa} \frac{B \theta}{r^{2}}
$$




\section{Inhomogeneous quantum vortex tangles}

4.5. Only the term in $\frac{\partial V}{\partial x} \frac{\partial L}{\partial x}$.

In this case equation (23) becomes:

$$
\frac{\partial L}{\partial t}=\alpha_{V} V L^{\frac{3}{2}}-\beta_{V} \kappa L^{2}+\frac{3}{2} L^{-\frac{1}{2}} \frac{\partial V}{\partial x} \frac{\partial L}{\partial x},
$$

and one obtains:

$$
L^{\prime}=\frac{6}{\alpha_{V} V^{2}}\left(\frac{\partial V}{\partial x}\right)^{2}=6 \frac{\theta^{2}}{\alpha_{V} r^{2}}
$$

4.6. Only the term in $\frac{\partial^{2} V}{\partial x^{2}}$.

In this case, one has:

$$
\frac{\partial L}{\partial t}=\alpha_{V} V L^{\frac{3}{2}}-\beta_{V} \kappa L^{2}+\frac{1}{2} \alpha_{V} L^{\frac{1}{2}} \frac{\partial^{2} V}{\partial x^{2}},
$$

obtaining:

$$
L^{\prime}=\frac{1}{V} \frac{\partial}{\partial x}\left(\frac{\partial V}{\partial x}\right)=2 \frac{\theta^{2}}{r^{2}} .
$$

The final result of the complete analysis of each term would be that the perturbation with respect to Vinen's solution would have the form:

$$
L^{\prime}=\frac{2}{\beta_{V} \kappa}\left(2 \alpha_{V}+2 \gamma_{1}-\gamma_{3}\right) \frac{B \theta}{r^{2}}+8 \frac{\gamma_{2}}{\beta_{V} \kappa^{2}} \frac{B^{2} \theta^{2}}{r^{4}}+12\left(\frac{D}{\beta_{V} \kappa}-1\right) \frac{\theta^{2}}{r^{2}}
$$

The parameters $\alpha_{V}$ and $\beta_{V}$ are known from steady-state homogeneous situations. Coefficients $\gamma_{1}, \gamma_{2}$ and $\gamma_{3}$ may be obtained and checked sperimentally as well as their thermodynamic consistency, which will be examined in future works.

\section{Conclusions.}

In this paper we have examined how inhomogeneities in $\mathbf{V}$ or $\mathbf{q}$ and $L$ may contribute to the dynamics of $L$ in inhomogeneous vortex tangles. This situation is found in radial flows, flows in convergent channel, or flows in entrance regions. In the previous paper $[22,23,25]$ we studied some aspects of this problem from a thermodynamic perspective, to examine the restrictions on the sign of some coefficients imposed by the second law of thermodynamics. Essentially, the terms in $D$ and $\alpha^{\prime \prime}$ in equation (3), plus other terms depending on temperature gradient that we have not considered here. In the present paper we have taken a different perspective, focused 


\section{Saluto, M.S. Mongiovì}

on the dynamical possibilities, rather than on their thermodynamic consistency, which could be examined in future works.

In this paper our strategy has been to start from Vinen equation with diffusion terms and adding to it two kinds of new non-local terms:

a) those coming from a second-order Taylor expansion of the vortex creation and destruction terms, in terms of the average vortex separation, given by $L^{-\frac{1}{2}}$;

b) other kinds of second-order terms with suitable dimensionality and satisfying tensorial requirements.

First, as a motivation to our analysis, we have considered the possible role of the heat flux gradient as additional sources of vortices, not considered in Vinen equation, and we have applied it to radial heat flow. Successively, we have made a systematic analysis of the physical consequences of the several non-local terms on the vortex line distribution in convergent (or divergent) channels.

The final result of this analysis is the perturbation $L^{\prime}$ of the Vinen's solution, which is obtained from equation (19) considering $\mathbf{V}$ and $\hat{u}$ collinear and having same direction, as in Figure 5. However in expression (19) some terms, i.e. those arising from scalar products between $\mathbf{V}$ (or $\mathbf{q}$ ) and $\nabla L$, could have different sign, because of the direction of the heat flux with respect to the gradient of $L$ and they will depend on whether heat flux goes to the right of Figure 6 or it goes to the left. This lead heat rectification in convergent or divergent channels.

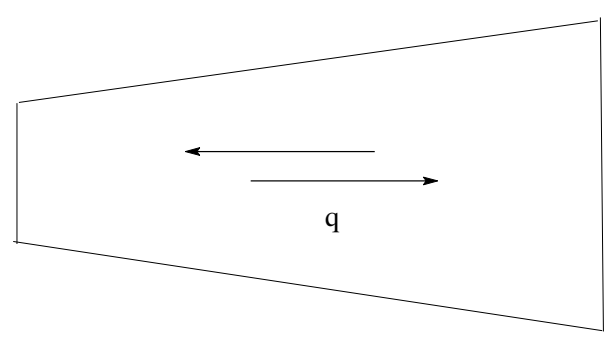

Figure 6. Heat flux along $x$-axis in a channel with varying transversal section. In steadystates, the change in the transversal area implies a corresponding change of the average heat flux across the section.

In particular, we can observe in Figure 3 or Figure 6 (where we have represented the longitudinal section of a channel) that the heat flux density $\mathbf{q}$ will be higher in the narrow transversal section than in the wide transversal section, since the total heat flow in the steady-state must be homogeneous. 


\section{Inhomogeneous quantum vortex tangles}

Finally, note that the results of this paper generalize those predicted by Vinen equation, according to which $L$ at a given point should depend only on the heat flux at that point, but not on the gradient nor the direction of the heat flux. A detailed comparison of the predicted perturbations with experimental results in convergent (or divergent) channel would allow us to check the theory.

\section{Acknowledgements.}

The authors acknowledge the support of the Università di Palermo (under Grant Nos. Fondi 60\% 2012) and the financial support of "National Group of Mathematical Physics" GNFM-INdAM. L.S. acknowledges the Università di Palermo for supporting her research stay at the Universitat Autònoma de Barcelona (March-June 2013 and January-July 2014) and the hospitality of the Departament de Física of the "Universitat Autònoma de Barcelona"

\section{REFERENCES}

1. P. Granieri, B. Baudouy, A. Four, F. Lentijo, A. Mapelli, P. Petagna, and D. Tommasini, Steady-state heat transfer through micro-channels in pressurized He II, AIP Conf. Proc., vol. 1434, pp. 231-238, 2011. DOI:10.1063/1.4706925.

2. J. Maurer and P. Tabeling, Local investigation of superfluid turbulence, EPL (Europhysics Letters), vol. 43, no. 1, p. 29, 1998.

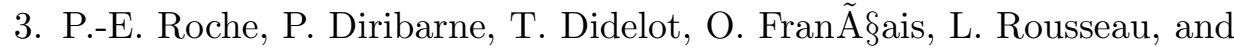
$\mathrm{H}$. Willaime, Vortex density spectrum of quantum turbulence, EPL ( $E u$ rophysics Letters), vol. 77, no. 6, p. 66002, 2007.

4. A. W. Baggaley, L. K. Sherwin, C. F. Barenghi, and Y. A. Sergeev, Thermally and mechanically driven quantum turbulence in helium II, Phys. Rev. B, vol. 86, p. 104501, 2012.

5. R. J. Donnelly, Quantized vortices in helium II. Cambridge: Cambridge University Press, 1991

6. C. F. Barenghi, R. J. Donnelly, and W. F. Vinen, Quantized Vortex Dynamics and Superfluid Turbulence. Berlin: Springer, 2001.

7. M.Tsubota, M.Kobayashi, and H. Takeuchi, Quantum hydrodynamics, Phys. Rep., vol. 522, pp. 191-238, 2012. DOI:10.1016/j.physrep.2012.09.007.

8. S. van Sciver, Helium cryogenics. Berlin: Springer, second ed., 2012.

9. S. K. Nemirovskii, Quantum turbulence: Theorethical and numerical problems, Phys. Rep., vol. 524, pp. 85-202, 2013. DOI:10.1016/j.physrep.2012.10.005. 


\section{Saluto, M.S. Mongiovì}

10. W. F. Vinen, Mutual friction in a heat current in liquid helium II III. theory of the mutual friction, Proc. Roy. Soc. London, vol. A240, pp. 493-515, 1957. DOI:10.1098/rspa.1957.0191.

11. J. Castiglione, P. J. Murphy, J. T. Tough, F. Mayot, and Y. Pomeau, Propagating and stationary superfluid turbulent fronts, Phys. B, vol. 100, pp. 575-595, 1995. DOI:10.1007/BF00751526.

12. J. F. Kafkalidis, G. Klinich III, and J. T. Tough, Superfluid turbulence in a nonuniform rectangular channel, Rep. Prog. Phys., vol. 50, p. 15909 (20 pages), 1994. DOI:10.1103/PhysRevB.50.15909.

13. G. Klinich III, J. F. Kafkalidis, and J. T. Tough, Superfluid Turbulence in Converging and Diverging Rectangular Channels, J. Low Temp. Phys., vol. 107, pp. 327-346, 1997. DOI:10.1007/BF02397461.

14. J. P. Murphy, J. Castiglione, and J. T. Tough, Superfluid turbulence in a nonuniform circular channel, J. Low Temp. Phys., vol. 92, pp. 307-334, 1993. DOI:10.1007/BF00682294.

15. J. F. Kafkalidis, G. Klinich III, and J. T. Tough, The vortex line density in nonuniform superfluid turbulence, Physica B, vol. 194-196, pp. 717718, 1994. DOI:10.1016/0921-4526(94)90688-2.

16. S. K. Nemirovskii, Diffusion of inhomogeneous vortex tangle and decay of superfluid turbulence, Phys. Rev. B, vol. 81, p. 64512 (10 pages), 2010. DOI:10.1103/PhysRevB.81.064512.

17. S. K. Nemirovskii, Propagation of a Turbulent Fronts in Quantum Fluids, J. Low Temp. Phys., vol. 162, pp. 347-353, 2011. DOI:10.1007/s10909-010-0252-x.

18. L. Kondaurova and S. K. Nemirovskii, Numerical study of decay of vortex tangles in superfluid helium at zero temperature, Phys. Rev. B, vol. 86, p. 134506 (13 pages), 2012. DOI:10.1103/PhysRevB.86.134506.

19. J. A. Geurst, Hydrodynamics of quantum turbulence in He II: Vinen's equation derived from energy and impulse of vortex tangle, Physica B, vol. 154, pp. 327-343, 1989. DOI:10.1016/0921-4526(89)90167-1.

20. J. A. Geurst, Hydrodynamic theory of superfluid turbulence in He II and Schwarz's vortex modelling, Physica A, vol. 183, pp. 279-303, 1992. http://EconPapers.repec.org/RePEc:eee:phsmap:v:183:y:1992:i:3:p:279303.

21. J. A. Geurst and H. van Beelen, Hydrodynamics of superfluid turbulence in He II: three-dimensional theory, Physica A, vol. 206, pp. 58-92, 1994. http://EconPapers.repec.org/RePEc:eee:phsmap:v:206:y:1994:i:1:p:5892.

22. M. S. Mongiovì and D. Jou, Thermodynamical derivation of a hydrodynamical model of inhomogeneous superfluid turbulence, Phys. Rev. B, vol. 75, p. 24507 (14 pages), 2007. DOI:10.1103/PhysRevB.75.024507. 


\section{Inhomogeneous quantum vortex tangles}

23. L. Saluto, M. S. Mongiovì, and D. Jou, Vortex diffusion and vortex-line hysteresis in radial quantum turbulence, Physica B, vol. 440C, pp. 99103, 2014. DOI:10.1016/j.physb.2014.01.041.

24. K. W. Schwarz, Three-dimensional vortex dynamics in superfluid He 4: Homogeneous superfluid turbulence, Phys. Rev. B, vol. 38, pp. 23982417, 1988. DOI:10.1103/PhysRevB.38.2398.

25. L. Saluto, D. Jou, and M. S. Mongiovì, Thermodynamic approach to vortex production and diffusion in inhomogeneous superfluid turbulence, Physica A, vol. 406, pp. 272-280, 2014. DOI:10.1016/j.physa.2014.03.062.

26. L. J. Campbell, Driven, dissipative superfluids: Radial counterflow of rotating ${ }^{4} \mathrm{He}$, Phys. Rev. B, vol. 36, pp. 6773-6781, 1987. DOI:10.1103/PhysRevB.36.6773.

27. M. S. Mongiovì and D. Jou, Generalization of Vinen's equation including transition to superfluid turbulence, J. Phys., vol. 17, pp. 4423-4440, 2005. http://stacks.iop.org/0953-8984/17/i=28/a=003.

28. L. Saluto, M. S. Mongiovì, and D. Jou, Longitudinal counterflow in turbulent liquid helium: velocity profile of the normal component, $Z$. Angew. Math. Phys., vol. 65, pp. 531-548, 2014. DOI:10.1007/s00033013-0372-7.

29. L. Saluto, Stationary heat flux profile in turbulent helium II in a semiinfinite cylindrical channel, in Bollettino di Matematica Pura e Applicata, vol. V, pp. 133-144, Aracne, 2012.

30. M. Sciacca, M. S. Mongiovì, and D. Jou, Alternative Vinen equation and its extension to rotating counterflow superfluid turbulence, Physica B, vol. 4038, pp. 2215-2224, 2008. DOI:10.1016/j.physb.2007.12.001.

31. K. P. Martin and J. T. Tough, Evolution of superfluid turbulence in thermal counterflow, Phys. Rev. B, vol. 27(5), pp. 2788-2799, 1983. DOI:10.1103/PhysRevB.27.2788. 